\title{
Rubella virus, Toxoplasma gondii and Treponema pallidum congenital infections among full term delivered women in an urban area of Tanzania: a call for improved antenatal care
}

\author{
Mariam M Mirambo ${ }^{1}$, Stephen E Mshana ${ }^{1}$, Uwe Gro $\beta^{2}$
}

1. Department of Microbiology and Immunology, Weill Bugando School of Medicine, Catholic University of Health and Allied Sciences, P.O. Box 1464, Mwanza, Tanzania.

2. Institute of Medical Microbiology, Gottingen University Medical Centre, Germany.

\section{Emails:}

MMM: mmmirambo@gmail.com

SEM: stephen72mshana@gmail.com

UG: ugross@gwdg.de

\begin{abstract}
Background: A significant proportion of newborns in the developing countries are born with congenital anomalies.

Objective: This study investigated congenital infections due to Rubella virus, Toxoplasma gondii, Treponema pallidum among presumed normal neonates from full term pregnant women in Mwanza, Tanzania.

Methods: Sera from mothers were tested for Treponema pallidum and Toxoplasma gondii infection while newborns from mothers with acute infections were tested for T. pallidum and T. gondii, and all newborns were tested for Rubella IgM antibodies.

Results: A total of 13/300 (4.3\%) mothers had T. pallidum antibodies with 3 of them having acute infection. Two (0.7 \%) of the newborns from mothers with acute infection were confirmed to have congenital syphilis. Regarding toxoplasmosis, 92/300 $(30.7 \%)$ mothers were IgG seropositive and 7 had borderline positivity, with only 1/99 (1\%) being IgM seropositive who delivered IgM seronegative neonate. Only 1/300 (0.3\%) newborn had rubella IgM antibodies indicating congenital rubella infection. Conclusion: Based on these results, it is estimated that in Mwanza city in every 100,000 live births about 300 and 600 newborns have congenital rubella and syphilis infections, respectively. Rubella virus and T. pallidum are likely to be among common causes of congenital infections in developing countries.
\end{abstract}

Keywords: Congenital infections, Mwanza, Tanzania.

DOI: https://dx.doi.org/10.4314/ahs.v19i2.8

Cite as: Mirambo MM, Mshana SE, Gro $\beta$ U. Rubella virus, Toxoplasma gondii and Treponema pallidum congenital infections among full term delivered women in an urban area of Tanzania: a call for improved antenatal care. Afri Health Sci.2019;19(2): 1858-1865. bttps://dx.doi. org/10.4314/abs.v19i2.8

\section{Background}

Congenital and perinatal infections are the leading cause of childhood morbidity and mortality particularly in low

\section{Corresponding author:}

Mariam M Mirambo,

Department of Microbiology and Immunology,

Weill Bugando School of Medicine,

P.O. Box 1464, Mwanza, Tanzania

Phone +255 754801 665, Fax: +255282502678

Email address: mmmirambo@gmail.com and middle-income countries (LMIC). Congenital infections acronymed as TORCH (Toxoplasma gondii (T. gondii); Other (e.g., Streptococcus agalactiae, Listeria spp.); Rubella virus; Cytomegalovirus (CMV) and Herpes simplex virus (HSV) proposed by Nahmias et al in 1970's has been recently expanded to include other infections acronymed as CHEAPTORCHES standing for $\mathrm{C}$ - Chickenpox and shingles; H - Hepatitis B, C, (D), E; E - Enterovirus; A - AIDS (HIV infection); P -Parvovirus B19; TORCH; E - Everything else sexually transmitted (Neisseria gonorrhea, Chlamydia trachomatis, Ureaplasma urealyticum, Human 
papillomavirus); S - Syphilis ${ }^{2}$. This expansion entails to incorporate a number of infections that can potentially cause congenital infections with serious consequences to the health of newborn soon after birth or later in life. Overwhelming evidence suggest that maternal infections are common in $\mathrm{LMIC}^{2-13}$ with limited information regarding congenital infections. Following a call by the World Health Organization (WHO) on the improvement on maternal and child health ${ }^{14}$, many high-income countries (HIC) embarked on improved health services to account for the consequences of infections which appears to be common worldwide. Despite the effort of different health authorities in LMIC, these infections are still the leading causes of prenatal and perinatal infections in these countries. The course of these infections depends on several factors such as mode of transmission, timing of infection in respect to gestational age, predilection of the pathogen, maturity of the fetal immune system and presence or absence of the maternal immunity at the time of infection ${ }^{15}$. Each of the pathogen can cause various clinical manifestations, however most of them might also lead to asymptomatic infection in the pregnant women and newborns. The WHO recommends antenatal and prenatal screening for most of these infections to prevent vertical transmission and possible consequences to the newborns. Maternal screening, treatment or vaccination where appropriate have been found to be effective strategies to significantly reduce the burden of these infections in $\mathrm{HIC}^{2}$. However, the burden is still high in LMIC due to the financial constraints and health policy priorities. Apart from including the screening of few of these pathogens such as human immunodeficiency virus (HIV) and T. pallidum in most of these countries, there is no monitoring of the effectiveness of screening programmes, and other pathogens such Rubellavirus and T. gondii are not routinely screened. In addition, there is paucity of data regarding the magnitude of these infections among newborns who appear normal at birth in most of the LMIC. Previous studies ${ }^{3,4,6,9}$ in the city of Mwanza have reported Rubella virus, T. gondii and T. pallidum to be common among pregnant women with limited data of infections of these pathogens in presumed normal newborns. This study was designed to estimate the magnitude of congenital Rubella virus, $T$. gondii and $T$. pallidum infections among presumed normal live neonates from full term pregnant women

\section{Methodology}

Study design, duration, study area and study population

A cross-sectional hospital-based study was conducted at Sekou Toure Regional Hospital (STH) and the Bugando Medical Centre (BMC) between January and May 2015. A total of 300 women (114 from the BMC and 186 from STH labor wards) were enrolled.

\section{Inclusion and exclusion criteria}

All women aged 18 years and above with normal deliveries who consented to participate in the study were enrolled. In order to establish the magnitude of congenital infections in normal live births, all neonates with obvious congenital defects at birth were excluded.

\section{Data and specimen collection}

Socio-demographic information such as age, residence, occupation, marital status, education, and other relevant clinical information such as gravidity and gestation age were collected using a pre-tested structured data collection tool. Paired samples (about $4 \mathrm{~mL}$ of mother's venous blood and $4 \mathrm{~mL}$ of cord blood) were collected aseptically in a plain vacutainer tubes (Becton Dickinson (BD), Nairobi, Kenya). All specimens were transported to the Bugando Medical Centre where sera were separated from the whole blood by centrifugation at 3,000 rpm for $5 \mathrm{~min}$ utes. Sera were numbered accordingly and kept at $-80^{\circ} \mathrm{C}$ until transportation to Germany for further analysis at the Institute of Medical Microbiology, Gottingen University Medical Centre, Germany.

\section{Diagnosis of congenital infection}

In order to establish congenital infections, different algorithms were followed for screening of these pathogens in the cord blood. T. gondii congenital infections was investigated by screening all neonates from mothers who tested positive for both T. gondii IgG and IgM antibodies. Congenital syphilis was established by testing neonates from mothers with recent $T$. pallidum infection while for congenital rubella infections all neonates were tested for Rubella IgM antibodies ${ }^{16-18}$.

\section{Laboratory analysis of the samples}

For the diagnosis of $T$. gondii infections ${ }^{17}$, sera were tested for anti-T. gondii antibodies using Enzyme linked Fluo- 
rescent Assay(ELFA) kits (VIDAS Toxo-IgG-II- (ELFA) and Toxo-IgM-ELFA for anti-T. gondii-specific IgG and IgM antibodies, respectively (bioMérieux, France). The cut-off values for detection of $\operatorname{IgG}$ antibodies were as follows: sera with $\mathrm{IgG}$ levels $<4 \mathrm{IU} / \mathrm{mL}$ were regarded as negative, $\mathrm{IgG}$ levels $4-<8 \mathrm{IU} / \mathrm{mL}$ were regarded as borderline and $\mathrm{IgG}$ levels $\geq 8 \mathrm{IU} / \mathrm{mL}$ were considered as positive. The cut-off values for detection of $\operatorname{IgM}$ were as follows: index value of $<0.55$ was considered as $\operatorname{IgM}$ negative, index value of $0.55-<0.65$ was borderline and index value of $\geq 0.65$ was termed as IgM positive. Rubella congenital infections was investigated using cord blood for the presence of specific rubella IgM antibodies $^{16,19}$. Detection was done by using AxSYM rubella virus IgM-microparticle enzyme immunoassay (MEIA) (Abbott, IL, USA); anti-rubella IgM of $>0.8$ was considered as positive and anti-rubella IgM of $<0.6$ was considered as negative and $\operatorname{IgM}$ value between $0.6-0.8$ was considered as borderline. The reversed syphilis algorithm diagnosis was employed in the detection of recent Treponema pallidum infection using Serodia Treponema pallidum particle assay (TPPA) (Fujirebio, Japan) and Venereal Disease Research Laboratory (VDRL) test (Omega Diagnostics, Germany $)^{18}$. All laboratory methods were performed according to manufacturer instructions.

\section{Data analysis}

All data were entered by Microsoft Office Excel 2007 and transferred to STATA version 12 (College Station, Texas, USA) for cleaning and analysis. Categorical variables were summarized as proportions and were analyzed using the Pearson's Chi-square test to observe the statistical differences among the various groups. Continuous variables were summarized as median with interquartile range or means with standard deviations. The differences in medians were statistically compared using Wilcoxon ranksum Mann-Whitney test. Univariate analysis and multivariate logistic regression models were performed to determine the predictors of T. gondii IgG seropositivity among pregnant women. Variables with p-value less than 0.2 on univariate analysis were fitted into the multivariate logistic regression analysis to establish independent predictors. A $P$ value of $<0.05$ at $95 \%$ confidence interval was considered statistically significant.

\section{Ethical considerations}

The study protocol was approved by the CUHAS/BMC Research Ethics Review Committee (CREC) with ethical clearance number CREC/043/2014. An informed written consent was sought from each enrolled woman.

\section{Results \\ Socio-demographic characteristics of the study par- ticipants}

The median age of the 300 enrolled women was 24 (IQR: 20.5-29.5) years while the mean gestation age at delivery was $38 \pm 1.44$ weeks. A significant proportion $124(41.3 \%)$ of participants were primigravida while 123 $(41.0 \%)$ were housewives (Table 1). On Wilcoxon ranksum Mann-Whitney test, primigravida women had significantly younger age compared to multigravida women (20, IQR: $18-22$ vs. 28 , IQR: 24.4-32 years, P <0.001). Regarding education level, the majority of these women $215(71.67 \%)$ had either no formal education or primary education. In addition, a significant proportion $72(24 \%)$ of these women reported to have been drinking unboiled water while $14(4.7 \%)$ reported to have been keeping cats at home (Table 1). 
Table1: Socio-demographic characteristics of the $\mathbf{3 0 0}$ full term delivered women in the city of Mwanza

\begin{tabular}{lcc}
\hline Study variable & Number of patients & Percent (\%)/Median IQR/Mean \pm SD \\
\hline Age (years) & 300 & $2420.5-29.5$ \\
\hline Gestation age at birth & 300 & $38 \pm 1.44$ \\
\hline Marital status & 262 & 87.3 \\
\hline Married & 38 & 12.7 \\
\hline Unmarried & 123 & \\
\hline Occupation & 22 & 41.0 \\
\hline Housewives & 101 & 7.3 \\
\hline Employed & 54 & 33.7 \\
\hline Small scale business & & 18.0 \\
\hline Peasant & 215 & \\
\hline Education level & 85 & 71.7 \\
\hline Primary/No formal & & 28.3 \\
\hline Secondary/tertiary & 124 & \\
\hline Gravidity & 176 & 41.3 \\
\hline Primigravida & & 58.7 \\
\hline Multigravida & 72 & \\
\hline Drinking unboiled water & 228 & 24.0 \\
\hline Yes & 14 & 76.0 \\
\hline No & 286 & 4.7 \\
\hline Keeping cats & & 95.3 \\
\hline Yes & & \\
\hline No & & \\
\hline
\end{tabular}

Prevalence of congenital infections and associated factors among full term delivered women in the city of Mwanza, Tanzania

Out of 300 enrolled women 13 (4.3\%) were TPPA positive indicating exposure to T. pallidum. Of these $13 \mathrm{TP}$ PA-positive women, 3 were VDRL positive indicating the overall prevalence of $1.0 \%$ of recent $T$. pallidum infection among women studied. Two (0.7\%) out 300 newborns were serologically confirmed to have congenital syphilis with no obvious symptoms observed, making about 600 cases of congenital syphilis infections in 100,000 live births. Increase in age was significantly associated with T. pallidum past infection (OR: 1.08, 95\% CI: 1.00-1.16, $\mathrm{P}=0.031$ ). Furthermore, the odds of having $T$. pallidum past infection was significantly higher among multigravida women than in primigravida women (OR: 3.76, 95\% CI: 1.06-13.27, $\mathrm{P}=0.040)$.
Regarding T. gondii, 92/300 (30.7\%) women were IgG seropositive with titers $\geq 8 \mathrm{IU} / \mathrm{mL}$ and 7 had borderline positivity i.e. titers between 4 and $8 \mathrm{IU} / \mathrm{ml}$. Out of 92 mothers who were $\operatorname{IgG}$ seropositive and 7 who had borderline titres, only 1/99 (1\%) was IgM seropositive; however, her newborn was not infected. On multivariate logistic regression analysis, being a small-scale business/ employed woman (OR=1.69, 95\%CI: 1.01-2.8, $\mathrm{P}=0.044)$ and drinking unboiled water $(\mathrm{OR}=1.78,95 \% \mathrm{CI}$ : 1.01-3.1, $\mathrm{P}=0.046)$ independently predicted $T$. gondii $\mathrm{IgG}$ seropositivity.

Rubella congenital infections (presence of IgM antibodies in cord blood) was detected in $1(0.3 \%)$ out of the 300 newborns tested, making about 300 cases of rubella congenital infections in 100,000 live births. 
Table2: Seroprevalence of $T$. gondii, Treponema pallidum and Rubella infections among mothers and full term delivered newborns in the city of Mwanza, Tanzania

\begin{tabular}{llllllll}
\hline & \multicolumn{2}{l}{ T. pallidum } & \multicolumn{2}{l}{ T. gondii } & \multicolumn{2}{l}{ Rubella } \\
\hline & Test & Positive & Test & Positive & & Positive \\
\hline Mothers $(\mathbf{n}=\mathbf{3 0 0})$ & TPPA & $13(4.3 \%)$ & IgG & $92(30.7 \%)$ & IgG & NT \\
\hline Mothers $(\mathbf{n}=\mathbf{3 0 0})$ & VDRL & $3(1.0 \%)$ & IgM & $1(0.3 \%)$ & IgM & NT \\
\hline Newborns(n=300) & VDRL & $2(0.7 \%)$ & IgM & $0(0.0 \%)$ & IgM & $1(0.3 \%)$ \\
\hline
\end{tabular}

NT = not tested

Table 3: Factors associated with $T$. gondii IgG seropositivity among women with full term delivery in the city of Mwanza, Tanzania

\begin{tabular}{|c|c|c|c|c|c|}
\hline \multirow[t]{3}{*}{ Characteristics } & \multirow{3}{*}{$\begin{array}{l}\text { IgG sero-positivity } \\
(\mathrm{N}, \%)\end{array}$} & \multicolumn{2}{|l|}{ Univariate } & \multicolumn{2}{|l|}{ Multivariate } \\
\hline & & OR $(95 \% \mathrm{Cl})$ & P value & OR (95\% Cl) & $P$ value \\
\hline & & & & & \\
\hline \multicolumn{6}{|l|}{ Marital status } \\
\hline Married & $79(30.2)$ & 1 & & & \\
\hline Unmarried & $13(34.2)$ & $\begin{array}{l}0.83(0.403- \\
1.70)\end{array}$ & 0.613 & & \\
\hline \multicolumn{6}{|l|}{ Gravidity } \\
\hline Primigravid & $33(26.6)$ & 1 & & & \\
\hline Multigravida & $59(33.5)$ & $1.39(0.83-2.3)$ & 0.202 & & \\
\hline \multicolumn{6}{|l|}{ Education } \\
\hline Secondary/Tertiary & $24(28.2)$ & 1 & & & \\
\hline Primary/No formal education & $68(31.6)$ & $0.85(0.48-1.47)$ & 0.566 & & \\
\hline \multicolumn{6}{|l|}{ Drinking unboiled water } \\
\hline No & $62(27.2)$ & 1 & & & \\
\hline Yes & $30(41.7)$ & 1.92(1.1-3.32) & 0.021 & $1.78(1.01-3.1)$ & 0.046 \\
\hline \multicolumn{6}{|l|}{ Keeping cat } \\
\hline No & $85(29.7)$ & 1 & & & \\
\hline Yes & $7(50.0)$ & $2.3(0.8-6.9)$ & 0.118 & $2.2(0.73-6.75)$ & 0.159 \\
\hline \multicolumn{6}{|l|}{ Occupation } \\
\hline Others & $47(26.5)$ & 1 & & & \\
\hline $\begin{array}{l}\text { Small scale } \\
\text { business/employed women }\end{array}$ & $45(36.6)$ & $1.58(0.97-2.6)$ & 0.171 & $1.69(1.01-2.8)$ & 0.044 \\
\hline \multicolumn{6}{|l|}{ HIV status } \\
\hline Negative & $11(25.6)$ & 1 & & & \\
\hline Positive & $2(14.3)$ & $\begin{array}{l}0.34(0.075- \\
1.58)\end{array}$ & 0.171 & $0.283(0.06-1.34)$ & 0.113 \\
\hline Unknown & $79(32.5)$ & $0.71(0.34-1.48)$ & 0.369 & $0.612(0.286-1.3)$ & 0.207 \\
\hline
\end{tabular}




\section{Discussion}

Prenatal and perinatal infections have been commonly associated with congenital anomalies and mortality particularly in low and middle income countries (LMIC) ${ }^{2}$. Many high income countries (HIC)have improved maternal and child health services to prevent the consequences of these infections following a call by the world health organization $(\mathrm{WHO})^{14}$. In LMIC efforts have been made, however effectiveness of screening for these infections during antenatal period and after birth have not been well emphasized. Here, we report the prevalence of selected infections with a potential risk for vertical transmis$\operatorname{sion}^{3,4,6,8,9}$ in the city of Mwanza among full term delivered women and their presumed normal newborns. The magnitude reported in this study among presumed normal newborns are considerably high calling for the need of considering these infections as an important agenda in the efforts to improve maternal and child health in LMICs.

In the current study, the prevalence of $T$. pallidum specific antibodies and its associated congenital infection was high signifying the importance of effective screening programmes. Our findings are in line with previous studies $^{20,21}$, which also reported high prevalence of congenital syphilis. Despite the current programme of screening T. pallidum infections during antenatal visits across the country, there is a need to consider re-screening during the course of pregnancy to account for the re-infections which has been documented to be $2.7 \%$. The current study confirms what has been reported previously that increase in age and multigravidity predict T. pallidum infections ${ }^{22,23}$. This could be explained by the fact that most of women enrolled in this study had advanced age which entails more exposure to $T$. pallidum infection as compared to young women.

Regarding T. gondii IgG seropositivity, the findings from the current study are comparable to the previous studies $^{3,24,25}$ indicating the endemicity of toxoplasmosis in Tanzania. In addition, the IgM seropositivity found in this study is also comparable with a previous study ${ }^{24}$ and lower compared to another report ${ }^{26}$. These findings suggest that there is possibility of the ongoing vertical transmission of $T$. gondii infections in this setting resulting to T. gondii associated adverse pregnancy outcomes. This calls for the need to introduce screening and treatment programme among women attending antenatal clinics in
Tanzania and other countries where $T$. gondii infection is endemic. In the current study, being formally employed or a small-scale business woman predicted $T$. gondii $\operatorname{IgG}$ seropositivity. This was also observed in a previous study ${ }^{3}$ in the same setting. It has been noted that living in town with reasonable income entails high socioeconomic status (SES). High SES can allow women to frequently afford meat dishes that can expose them to $T$. gondii infections $^{27,28}$.

Concerning rubella congenital infection, the findings from this study are comparable with previous studies in Tanzania and Sudan ${ }^{29,30}$. Rubella virus is endemic in Tanzania and other African countries with a significant proportion of women at high risk of contracting primary rubella virus infection that can lead to vertical transmission ${ }^{4,31}$. This was confirmed in the previous study which reported a significant number of pregnant women had acute rubella virus infection hence a potential risk to transmit it to the fetuses ${ }^{6}$. This calls for the need to emphasize on the antenatal screening and vaccination of child bearing women as suggested previously ${ }^{32}$.

Overall the current study highlights the importance of effective antenatal screening of these infections which will improve maternal and child health. Despite the inclusion of $T$. pallidum in antenatal screening, there is a paramount need of emphasizing re-screening during the course of pregnancy and / or at delivery. Being common in Tanzania T. gondii screening and treatment will improve antenatal care services across the country. In case of rubella, despite a strategy of vaccinating children, there is a need to consider additional strategy of vaccinating child bearing aged women in developing countries.

\section{Competing interest}

Authors declare that they have no competing interest. All authors have read and approve the final version of the manuscript to be submitted

\section{Authors' contributions}

All authors participated in the study design. MMM and SEM participated in the collection of the samples and clinical data. UG did laboratory analysis of all samples. MMM and SEM analysed and interpreted the data. MMM wrote the first draft of the manuscript. SEM and UG did critical review of the manuscript. All authors read and approved the final version of the manuscript. 


\section{Acknowledgements}

The authors would like to acknowledge the technical support provided by all staff at the Bugando Medical Centre, Sekou Toure Regional Hospital labour wards, and the Göttingen serology laboratory for their technical support. The study was supported by the Catholic University of Health and Allied Sciences and The Institute of Medical Microbiology, Göttingen University Medical Centre, Germany.

\section{References}

1. Nahmias AJ, Walls KW, Stewart JA, Herrmann KL, Flynt WJ: The ToRCH complex-perinatal infections associated with toxoplasma and rubella, cytomegol-and herpes simplex viruses. Pediatric Research 1971, 5(8):405-406.

2. Madrid L, Varo R, Sitoe A, Bassat Q: Congenital and perinatally-acquired infections in resource-constrained settings. Expert review of anti-infective therapy 2016, 14(9):845-861.

3. Mwambe B, Mshana SE, Kidenya BR, Massinde AN, Mazigo HD, Michael D, Majinge C, Groß U: Sero-prevalence and factors associated with Toxoplasma gondii infection among pregnant women attending antenatal care in Mwanza, Tanzania. Parasit Vectors 2013, 6:222.

4. Mwambe B, Mirambo MM, Mshana SE, Massinde AN, Kidenya BR, Michael D, Morona D, Majinge C, Groß $\mathrm{U}$ : Sero-positivity rate of rubella and associated factors among pregnant women attending antenatal care in Mwanza, Tanzania. BMC pregnancy and childbirth 2014, 14(1):95.

5. Mirambo MM, Mbena PB, Mushi MF, Mtebe M, Moremi N, Seni J, Mshana SE: Prevalence of Hepatitis B surface antigen among pregnant women attending antenatal clinic at Nyamagana District Hospital Mwanza, Tanzania. Tanzania Journal of Health Research 2016, 18(1).

6. Mirambo MM, Chibwe E, Mushi MF, Majigo M, Mshana SE: Cytomegalovirus, Parvovirus B19 and Rubella Co-infection among Pregnant Women Attending Antenatal Clinics in Mwanza City: The Need to be considered in Tanzanian Antenatal Care Package. Epidemiology: Open Access 2016, 2016.

7. Mhalu F, Haukenes G: Prevalence of cytomegalovirus antibody in pregnant women, AIDS patients and STD patients in Dar es Salaam. AIDS 1990, 4(12):1294.

8. Lulandala L, Mirambo MM, Matovelo D, Gumodoka B, Mshana SE: Acute Rubella Virus Infection among Women with Spontaneous Abortion in Mwanza City, Tanzania.
Journal of Clinical \& Diagnostic Research. 2017, 11(3).

9. Lawi JD, Mirambo MM, Magoma M, Mushi MF, Jaka HM, Gumodoka B, Mshana SE: Sero-conversion rate of Syphilis and HIV among pregnant women attending antenatal clinic in Tanzania: a need for re-screening at delivery. BMC Pregnancy and Childbirth. 2015, 15(1):3.

10. Simpore J, Savadogo A, Ilboudo D, Nadambega MC, Esposito M, Yara J, Pignatelli S, Pietra V, Musumeci S: Toxoplasma gondii, HCV, and HBV seroprevalence and co-infection among HIV-positive and-negative pregnant women in Burkina Faso. Journal of Medical Virology. 2006, 78(6):730-733.

11. Deperthes BD, Meheus A, O'Reilly K, Broutet N: Maternal and congenital syphilis programmes: case studies in Bolivia, Kenya and South Africa. Bulletin of the World Health Organization. 2004, 82(6):410-416.

12. Schulz K, Cates W, O'Mara P: Pregnancy loss, infant death, and suffering: legacy of syphilis and gonorrhoea in Africa. Sexually Transmitted Infections. 1987, 63(5):320-325.

13. Elnahas A, Gerais AS, Elbashir MI, Eldien ES, Adam I: Toxoplasmosis in pregnant Sudanese women. Saudi Medical Journal. 2003, 24(8):868-870.

14. WHO: The World Health Report 2005: Make every mother and child count: World Health Organization; 2005.

15. Menson E, Lyall H: Clinical presentation of congenital viral infections. Current Paediatrics. 2005, 15(2):163-170. 16. Chapter C: 15 (2012): Congenital Rubella Syndrome. Manual for the Surveillance of Vaccine-Preventable Diseases.

17. Control CfD, Prevention: DPDx: laboratory identification of parasites of Public Health Concern. In.; 1998. 18. Control CfD, Prevention: Syphilis testing algorithms using treponemal tests for initial screening--four laboratories, New York City, 2005-2006. MMWR Morbidity and Mortality Weekly Report. 2008, 57(32):872.

19. Marymont Jr JH, Herrmann KL: Rubella TestingAn Overview. Laboratory Medicine. 1982, 13(2):83-91.

20. Southwick KL, Blanco S, Santander A, Estenssoro M, Torrico F, Seoane G, Brady W, Fears M, Lewis J, Pope V: Maternal and congenital syphilis in Bolivia, 1996: prevalence and risk factors. Bulletin of the World Health Organization. 2001, 79(1):33-42.

21. McDermott J, Steketee R, Larsen S, Wirima J: Syphilis-associatedperinatal and infant mortality in rural Malawi. Bulletin of the World Health Organization. 1993, 71(6):773. 22. Endris M, Deressa T, Belyhun Y, Moges F: Se- 
roprevalence of syphilis and human immunodeficiency virus infections among pregnant women who attend the University of Gondar teaching hospital, Northwest Ethiopia: a cross sectional study. BMC Infectious Diseases. 2015, 15(1):111.

23. Jindal N, Arora U, Singh S, Devi B: Prevalence of sexually transmitted infections (HIV, hepatitis B, herpes simplex type 2 and syphilis) among asymptomatic pregnant women. The Journal of Obstetrics and Gynecology of India. 2012, 62(2):158-161.

24. Doehring E, Reiter-Owona I, Bauer O, Kaisi M, Hlobil H, Quade G, Hamudu NA, Seitz HM: Toxoplasma gondii antibodies in pregnant women and their newborns in Dar es Salaam, Tanzania. The American Journal of Tropical Medicine and Hygiene. 1995, 52(6):546-548.

25. Machumi I, Mirambo MM, Ruganuza D, Rambau P, Massinde AN, Kihunrwa A, Mshana SE, Moronac D: Factors Associated With Toxoplasma gondii IgG and IgM Antibodies, and Placental Histopathological Changes Among Women With Spontaneous Abortion in Mwanza City, Tanzania. Health Research Journal. 2017:86.

26. Guerina NG, Hsu H-W, Meissner HC, Maguire JH, Lynfield R, Stechenberg B, Abroms I, Pasternack MS, Hoff R, Eaton RB: Neonatal serologic screening and early treatment for congenital Toxoplasma gondii infection. New England Journal of Medicine. 1994, 330(26):1858-1863.
27. Ahmed HJ, Mohammed HH, Yusuf MW, Ahmed SF, Huldt G: Human toxoplasmosis in Somalia. Prevalence of Toxoplasma antibodies in a village in the lower Scebelli region and in Mogadishu. Transactions of the Royal Society of Tropical Medicine and Hygiene. 1988, 82(2):330-332.

28. Sroka J, Wójcik-Fatla A, Szymanska J, Dutkiewicz J, Zajac V, Zwolinski J: The occurrence of Toxoplasma gondii infection in people and animals from rural environment of Lublin region-estimate of potential role of water as a source of infection. Annals of Agricultural and Environmental Medicine. 2010, 17(1):125-132.

29. Adam O, Ali AK, Hübschen JM, Muller CP: Identification of congenital rubella syndrome in Sudan. BMC infectious diseases. 2014, 14(1):305.

30. Mirambo MM, Aboud S, Mushi MF, Seugendo M, Majigo M, Groß U, Mshana SE: Serological evidence of acute rubella infection among under-fives in Mwanza: a threat to increasing rates of congenital rubella syndrome in Tanzania. Italian Journal of Pediatrics. 2016, 42(1):1.

31. Mirambo MM, Majigo M, Aboud S, Groß U, Mshana SE: Serological makers of rubella infection in Africa in the pre vaccination era: a systematic review. BMC Research Notes. 2015, 8(1):716.

32. Mirambo MM, Hokororo A, Mrutu NB, Majigo M, Aboud S, Gross U, Mshana SE: Strategic immunization to control rubella and congenital rubella syndrome in Tanzania. CUHAS/PB/001, 2016:1-6. 